\title{
Piezo2-peripheral baroreceptor channel expressed in select neurons of the mouse brain: a putative mechanism for synchronizing neural networks by transducing intracranial pressure pulses
}

\author{
Jigong Wang ${ }^{1}$, Owen P. Hamill ${ }^{1, *}$ \\ ${ }^{1}$ Department of Neuroscience, Cell Biology and Anatomy, University of Texas Medical Branch, Galveston, TX 77555, USA \\ *Correspondence: ohamill@utmb.edu (Owen P. Hamill)
}

\section{DOI:10.31083/j.jin2004085}

This is an open access article under the CC BY 4.0 license (https://creativecommons.org/licenses/by/4.0/).

Submitted: 9 September 2021 Revised: 28 October 2021 Accepted: 28 October 2021 Published: 30 December 2021

\begin{abstract}
Here we use immunohistochemistry to examine the expression of Piezo2 in neurons of the mouse dorsal root ganglia and brain. Whereas Piezo2 is expressed in the large majority $(\geq 90 \%)$ of dorsal root ganglia neurons, Piezo2 expression is restricted to select neuron types in specific brain regions, including neocortical and hippocampal pyramidal neurons, cerebellar Purkinje cells and mitral cells of the olfactory bulb. Given the well-established role of Piezo2 as a low-threshold pressure sensor (i.e., $\leq 5 \mathrm{mmHg}$ ) in peripheral mechanosensation, including the regulation of breathing and blood pressure, its expression in central neurons has interesting implications. In particular, we hypothesize that Piezo2 provides neurons with an intrinsic resonance that promotes their entrainment by the normal intracranial pressure pulses $(\sim 5 \mathrm{mmHg})$ associated with breathing and cardiac cycles. The pressure-induced change in neural activity need only be very subtle to increase, for example, the robustness of respiration-entrained oscillations reported previously in widely distributed neuronal networks in both rodent and human brains. This idea of a "global brain rhythm" first arose from the effect of nasal airflow in activating mechanosensitive olfactory sensory neurons, which then synaptically entrain mitral cells within the olfactory bulb and through their projections, neural networks in other brain regions, including the hippocampus and neocortex. Our proposed, non-synaptic, intrinsic mechanism, where Piezo2 tracks the highly predictable and "metronome-like" intracranial pressure pulses - to date generally considered epiphenomena-would have the advantage that a physical force rapidly transmitted throughout the brain also contributes to this synchronization.
\end{abstract}

Keywords

Piezo2; Intracranial pressure; Neocortex; Hippocampus; Purkinje cells; Mitral cells; Olfactory bulb; Neural network entrainment; Clobal brain clock

Epiphenomena seldom remain epiphenomena for long; they are the raw material for evolutionary advances [1].

\section{Introduction}

Piezo2 is a pressure-activated channel expressed in peripheral neurons and known to play key roles in peripheral mechanosensation [2-4] including the regulation of arterial blood pressure [5] and breathing [6]. In comparison, the expression and possible function of Piezo2 in the brain remains relatively unexplored. One group using RT-PCR [7] and Western blotting [8] has reported Piezo2 expression in rat neocortical and hippocampal tissues and shown that this expression increases in response to repetitive mechanical (i.e., blast) injury. These findings raise the intriguing possibility that a sudden mechanical over activation of Piezo 2 channels caused by a concussion or mild traumatic brain injury (TBI) might contribute to the rapid and often reversible disruptions in brain functions, including loss of consciousness and memory. However, Piezo2 gene/protein expression measurement in brain tissue alone does not discriminate between expression in specific neurons and/or non-neuronal cell types (e.g., glial and vascular cells). We were further motivated to make this distinction based on a patch-clamp study of mouse brain slices in which pressure-activated single-channel currents were recorded in neocortical and hippocampal pyramidal neurons [9]. In the same study, pressure activation of only a single channel current was shown to trigger action potentials in the whole neuron and with stronger channel activation, high-frequency spiking [9]. Although the channel protein was not identified at the time, it was noted that the currents displayed "Piezo-like" single-channel conductance and gating characteristics [9]. Therefore, we have used a custom generated anti-PIEZO2 antibody $(\mathrm{Ab})$ and immunohistochemistry (IHC) to investigate the expression of Piezo2 in mouse brain slices. We were particularly interested in determining whether Piezo2 shows nonspecific, low-level expression, which could be functionally inconsequential, or instead is expressed in highly select cell types, similar to functionally critical voltage- and transmitter-gated channel subunits expressed in the brain. Recently, another group, using two different anti-PIEZO2 Abs, reported Piezo2 expression in rat peripheral and central neurons [10]. 


\section{Materials and methods}

\subsection{Isolation of mouse dorsal root ganglia and brains}

All experimental protocols were approved by the Animal Care and Use Committee at the University Texas Medical Branch (UTMB) and are in accordance with the NIH Guide for the Care and Use of Laboratory Animals. Young adult (20-25 g, $\sim 10$ weeks old) male C57BL/6J ( $\mathrm{n}=3$ ) or GAD67GFP ( $\mathrm{n}=3$ ) mice (Jackson Laboratory, Bar Harbor, Maine, USA). No differences were noted in the Ab staining patterns in the brains and dorsal root ganglia (DRG) from the two strains of mice. Mice were deeply anesthetized with 5\% isoflurane in oxygen. After exposing the pleural cavity, perfused through the left ventricle first with the cold heparinized saline aorta and then with $4 \%$ paraformaldehyde in $0.1 \mathrm{M}$ phosphate-buffered saline (PBS). Their DRG and brains were rapidly removed and stored in the same fixative overnight. Subsequently, the brains and DRG were dehydrated through an ethanol series/xylene embedded in paraffin, and $10 \mu \mathrm{m}$ sagittal slices were cut. Human red blood cells were collected in the fixative, pelleted, dehydrated, and embedded in paraffin for slicing.

\subsection{Antibody generation}

The anti-PIEZO2 Ab was custom generated by Proteintech (Chicago, Il, USA) against a peptide fragment overexpressed from a cloned fragment of the human PIEZO2 (pF1KE0043) that we purchased from Kazusa DNA. Research Institute (Chiba, Japan). This vector is suitable for expressing tag-free proteins in E. coli cells and in vitro proteinexpression systems driven by T7 RNA polymerase. The antigen (protein epitope signature tag (PrEST)) was a 188 amino acid peptide corresponding to amino acids 2440-2628 located at the C-terminus of the human PIEZO2 isoform (NP_071351.2) corresponding to amino acids 2512-2700 of the mouse Piezo2 isoform (NP_001034574.4). The plasmid insert was sequenced to ensure that the correct PrEST sequence was cloned. The size of the resulting recombinant protein (including the specific PrEST) was checked to ensure that the correct antigen was produced and purified. The rabbit $\mathrm{Ab}$ was affinity purified using an antigen-bound column. To control cross-reactivity, the affinity-purified Ab was tested for sensitivity and specificity on protein arrays consisting of glass slides with spotted PrEST fragments, including PIEZO1 and PIEZO2 fragments.

A primary aim in selecting the specific PIEZO2 PrEST was to generate an anti-PIEZO2 Ab with maximum binding affinity for the native mouse Piezo2 protein still embedded in the membrane (i.e., with its tertiary structure retained). We chose the C-terminal 188 amino acid peptide that encompasses part of the extracellular domain that contributes to Piezo2 pressure sensitivity [11, 12]. Another reason for this choice was that it should generate an $\mathrm{Ab}$ with high specificity for the mouse Piezo2 protein rather than, or in addition to, its paralog, Piezo1. The human PIEZO2 PrEST did display relatively high amino acid identity (i.e., 84\%, with 158 out of 188 residues identical) for the corresponding region (2512-2700) of the mouse Piezo2 isoform (NP_001034574.4) but relatively low identity (i.e., $34.6 \%$ with 65 out of 188 residue identity) with the corresponding region (3226-3414) of the mouse Piezo1 isoform (NP_001032375.1). Finally, the PrEST we chose, coincidently as it turned out, also encompasses the shorter C-terminal peptide (122 amino acids) of the corresponding amino acids 2446-2568 of PIEZO2 used to generate the commercial anti-PIEZO2 $\mathrm{Ab}$ (Atlas antibodies, HPA015986) ${ }^{1}$.

\subsection{Detecting Ab immunoreactivity}

The Piezo 2 immunoreactivity was detected using the antiPIEZO2 $\mathrm{Ab}$ at a 1:600 dilution for 60 minutes. The brain and DRG tissues were processed using the Dako Autostainer. The secondary Ab used was biotinylated goat anti-rabbit IgG (Vector Labs, Burlington, CA, \#BA-1000) at 1:200 for 30 minutes, followed by Dako LSAB2 streptavidin-HRP (\#SA5704) for 30 minutes. Slides were developed with Dako DAB chromagen (\#K3468) for 5 minutes and counterstained with Harris Hematoxylin for one minute. Imaging was carried out under bright field Olympus Bx51 microscope with Olympus DP imaging software. Because DAB staining does not fulfill the Beer-Lambert law, it is not stoichiometric [13], and staining intensity will not be related to the number of antigen molecules. Therefore, quantitative measurement of expression level was not possible. DRG and brain slices were viewed, and $\mathrm{Ab}$ stained cells were counted in high magnified (typically $40 \times$ objective) brain sections. Cells stained brown were counted as positive, with brown staining generally seen as all-or-none in which adjacent different cells types could be judged positive or negative. Negative controls made for the same cell types were compared in slices from the same $\mathrm{DRG} /$ brain region in which the primary Ab was omitted. For each experiment, where images of different slices were used for cell counting, the images were acquired with identical settings for detector gain and exposure time (i.e., 40×, $0.8 \mathrm{nu}$ merical aperture at $1024 \times 1024$ Pixel resolution).

\subsection{Data analysis and reproducibility}

Results were based on studies of six mice in which typically 3-4 slices of DRG and brains were examined. Only slices from mice that showed neurons with well-preserved morphology (i.e., when observed under high magnification) were selected for analysis. Our analysis focused on Piezo 2 expression in brain regions where neuron types were unequivocally identifiable based on their characteristic morphology and layered arrangement. As it turned out, pyramidal neurons within the neocortex and hippocampus, Purkinje cells (PC) in the cerebellar cortex and mitral cells (MC) of the olfactory bulb (OB) expressed Piezo2. In contrast, the more abundant granule cells in the dentate gyrus, cerebellar cortex, and $\mathrm{OB}$ were absent. We considered these yes/no patterns of

\footnotetext{
1 https://www.atlasantibodies.com/api/print_datasheet/HPA015986. pdf
} 

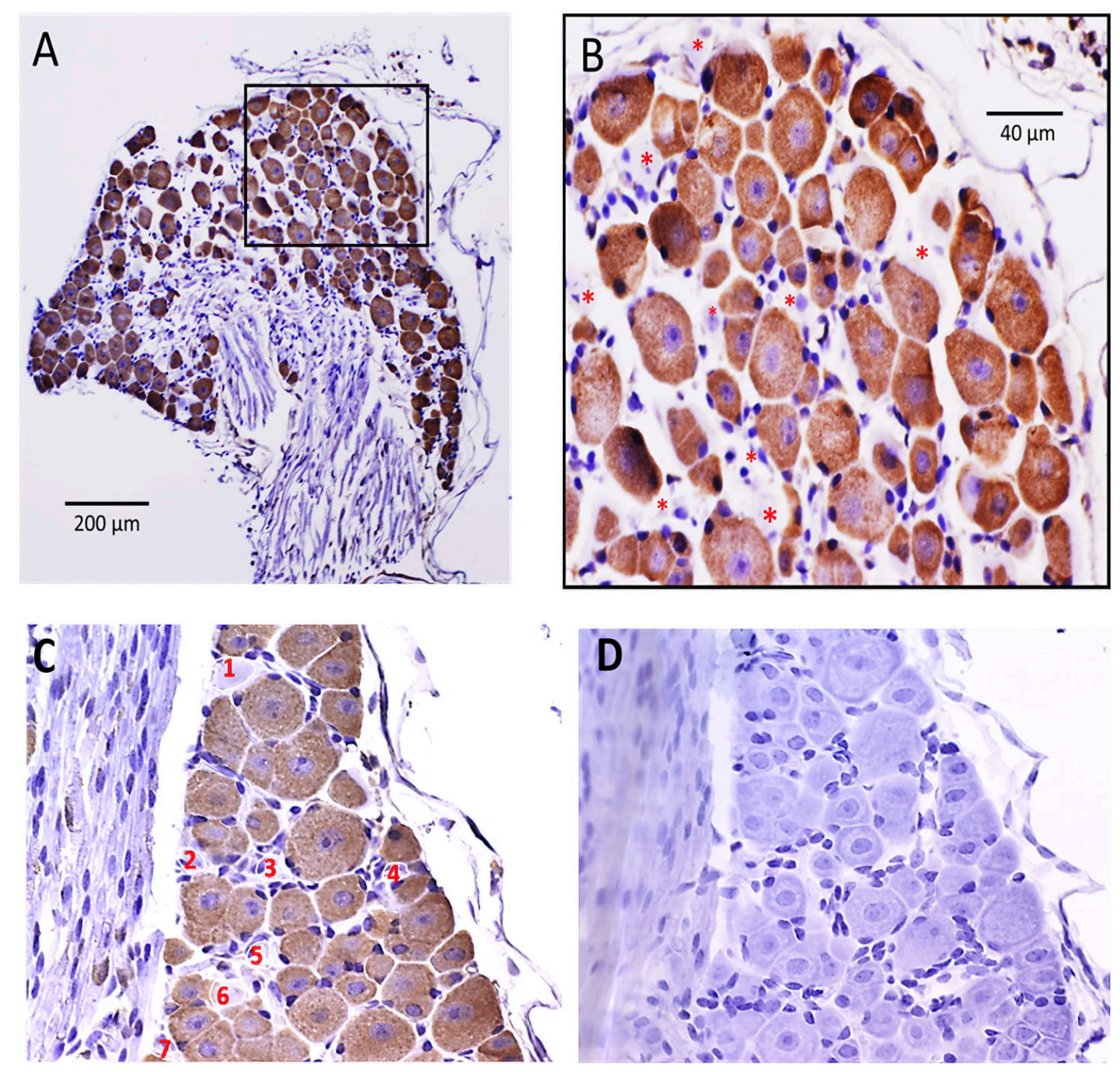

Fig. 1. Immunohistochemical localization of Piezo2 in mouse DRG. (A) The anti-PIEZO2 Ab stained most DRG neurons independent of soma size (range $10-50 \mu \mathrm{m}$ in diameter). (B) Higher magnification image of the section enclosed within the square in $1 \mathrm{~A}$ shows that of the $\sim 90$ neurons present, only 9 neurons (i.e., indicated by red asterisks) showed no Ab staining, and these appeared to be smaller diameter neurons. The numerous small blue structures (i.e., stained by the nuclear stain hematoxylin) around the perimeter of each neuron represent the cell bodies of satellite glial cells (SGCs) that include their cell nucleus and very thin cytoplasmic processes that ensheath the neurons. The SGCs showed no Ab staining, but this could be an issue of detection rather than a lack of Piezo2 expression. (C) Another DRG slice from a different mouse showed a similar pattern of staining most DRG neurons. (D) An adjacent slice treated with the same IHC protocol except without the anti-PIEZO2 Ab present.

Piezo2 expression reproducible if the same pattern was replicated in brain slices from at least 2 different mice. Because relative counts of stained/unstained cell types/morphologies measured in brain slices from different mice appeared very similar, counts were combined.

\section{Results \\ 3.1 Testing the sensitivity and specificity of the Anti-PIEZO2 Ab}

The critical requirement for our $\mathrm{Ab}$ generated against a human PIEZO2 peptide was high sensitivity and high selectivity in detecting the endogenous expression of the mouse ortholog Piezo2 but not its mouse paralog Piezo1. The lat- ter was important because Piezo1 is expressed in both mouse peripheral DRG $[14,15]$ and central neurons [16-18]. We used mouse DRG sensory neurons to confirm Ab sensitivity, where Piezo2 was first identified [2]. As indicated in Fig. $1 \mathrm{~A}, \mathrm{~B}$, the $\mathrm{Ab}$ stained the large majority of mouse DRG neurons $(\sim 90 \%)$, including the smallest $(\sim 10 \mu \mathrm{m})$ and largest $(\sim 50 \mu \mathrm{m})$ diameter neurons. This near pan expression in rodent DRG neurons is in line with recent ISH studies [14, 19], single-cell RNA-sequencing (scRNA-seq) $[20,21]$ and IHC studies [10] (see also Supplementary Fig. 1) but differs somewhat from the lower expression ( $\leq 60 \%$ of neurons) reported in the very first mouse DRG studies [2, 22]. Fig. 1C,D 

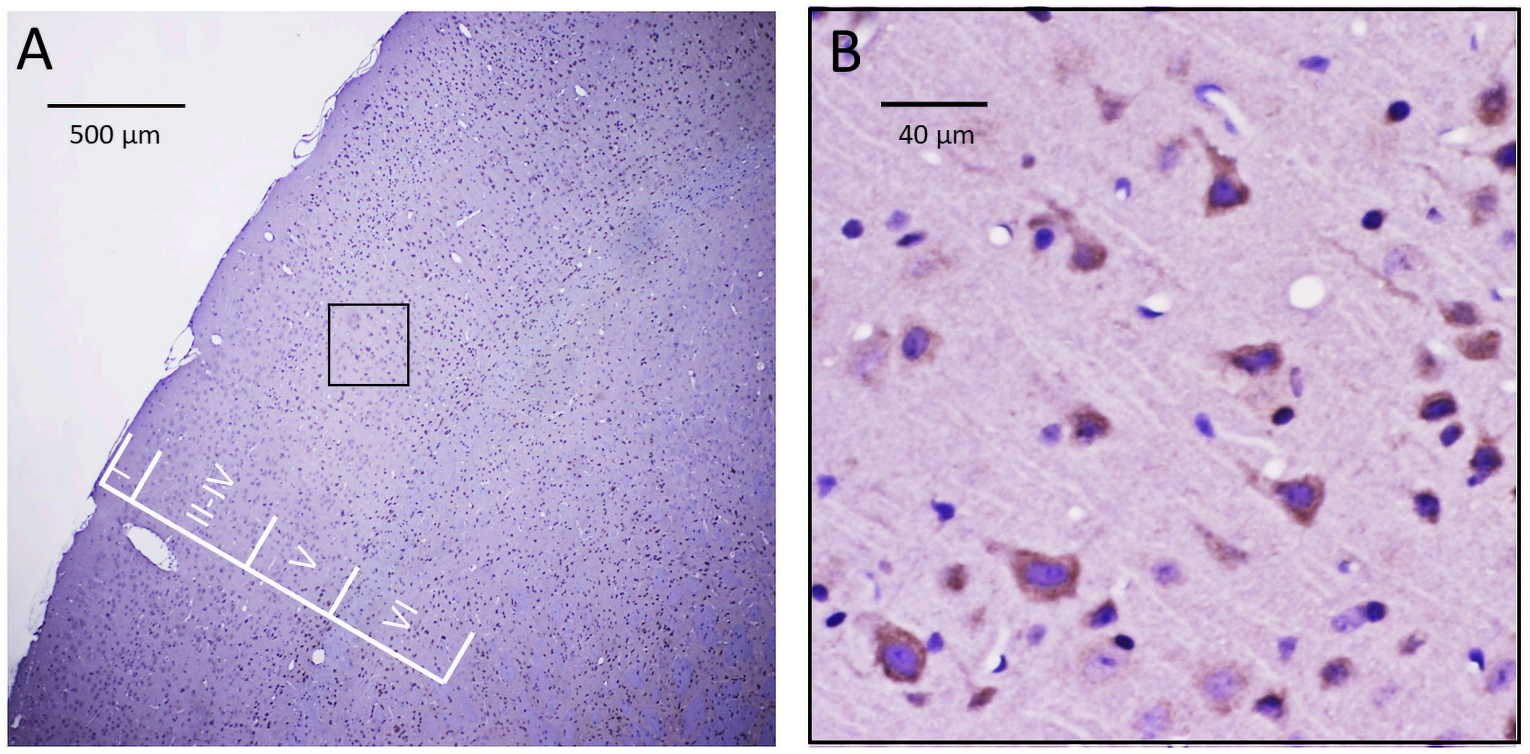

Fig. 2. Immunohistochemical localization of Piezo2 in a sagittal slice of mouse neocortex. (A) The anti-PIEZO2 Ab stained many cells throughout layers II-VI of the neocortex with minimal staining of cells in layer I. (B) The selected region in A (i.e., within the black box) representing a region within layer $\mathrm{V}$ is shown at higher magnification and indicates the most commonly stained cells displayed a pyramidal-shaped cell body with a thick process directed towards the pia (i.e., consistent with the apical dendrite of a pyramidal neuron). In contrast, there was less clear evidence of the staining of multipolar cells indicative of either stellate neurons or astrocytes.
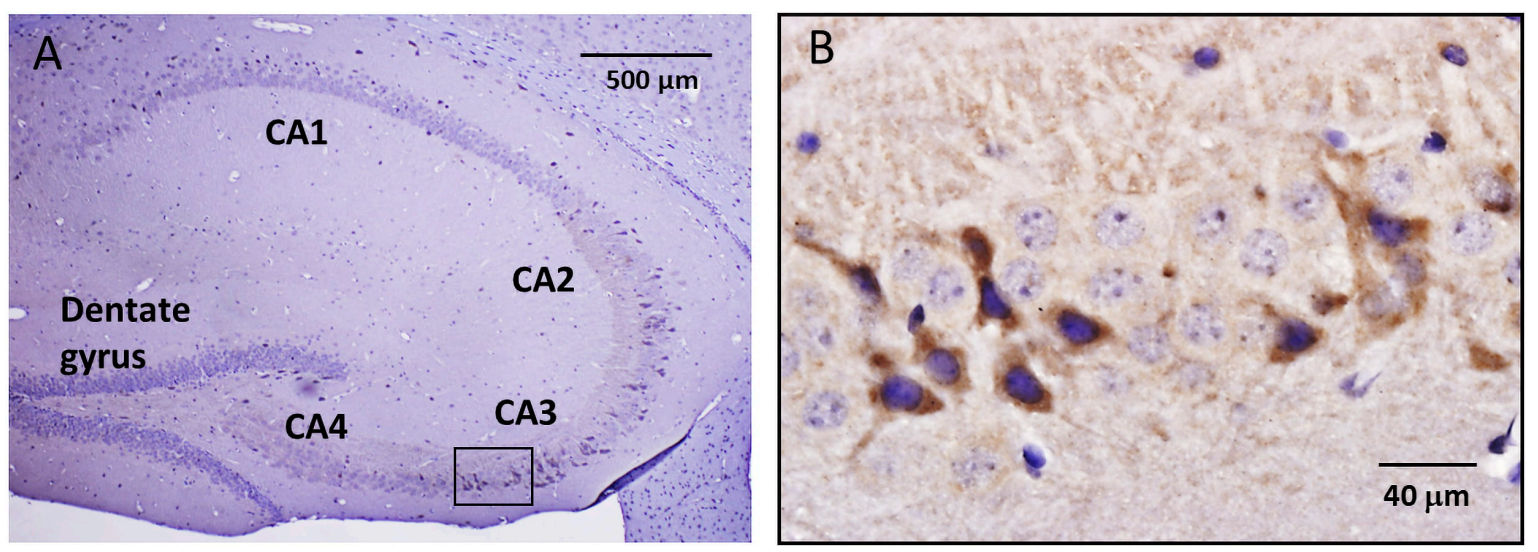

Fig. 3. Immunohistochemical localization of Piezo2 in mouse hippocampus. (A) Low magnification image of a hippocampal sagittal slice showing distinct divisions, including the dentate gyrus (DG) and CA4-CA1 regions. (B) The selected region in A (i.e., within the black box) that involves part of the CA3 region is shown at higher magnification and indicates at least 11 heavily stained cells, some of which displayed a distinct pyramidal-shaped cell body and a single prominent process. Within the same region, $\sim 25$ cells were stained blue by hematoxylin but showed no Ab staining. This all-or-none staining is at least consistent with the idea of a stochastic on-off switch controlling neuron type-specific Piezo2 expression.

demonstrates the lack of nonspecific staining by the secondary antibody using the same IHC protocol but without the anti-PIEZO2 $\mathrm{Ab}$. A further test for the anti-PIEZO2 Ab specificity was its lack of staining/cross-reactivity with the endogenous PIEZO1 expressed in human red blood cells [23].

\subsection{The Ab selectively stains pyramidal-like neurons in the mouse neocortex and hippocampus}

Using brain slices from the same mice that the DRG were isolated and following identical IHC protocols, we examined the expression of Piezo2 in different brain regions. In particular, the $\mathrm{Ab}$ stained many cells in the grey matter of the neocortex, most notably, cells spanning across layers $\mathrm{V}$ and VI (Fig. 2A). Examination at high magnification indicated that the stained cells were mostly pyramidal-like according to their triangular-shaped cell body and prominent unitary (apical) dendrite oriented perpendicular to the cortical layers and directed towards the pia (Fig. 2B). In contrast, we saw a much lower abundance of stained multipolar cells or cells with no processes, which may have represented stellate interneurons, astrocytes or even pyramidal neurons upon 3-D reconstruction (not carried out here). Counting these morphological types across different neocortical regions within 

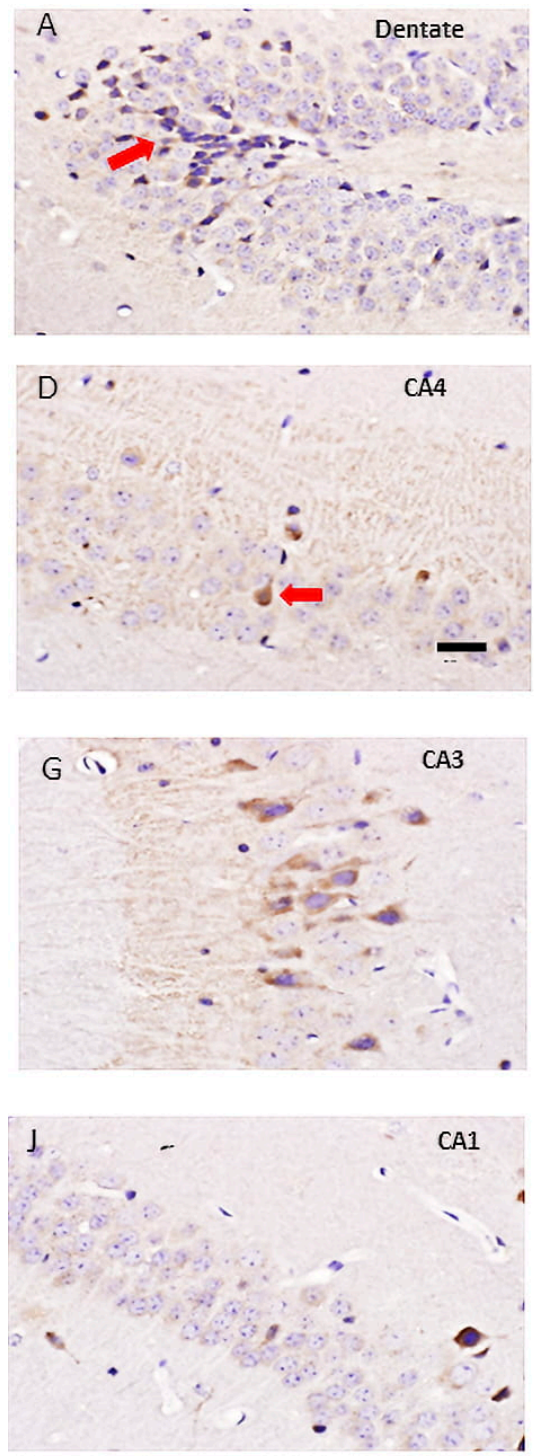
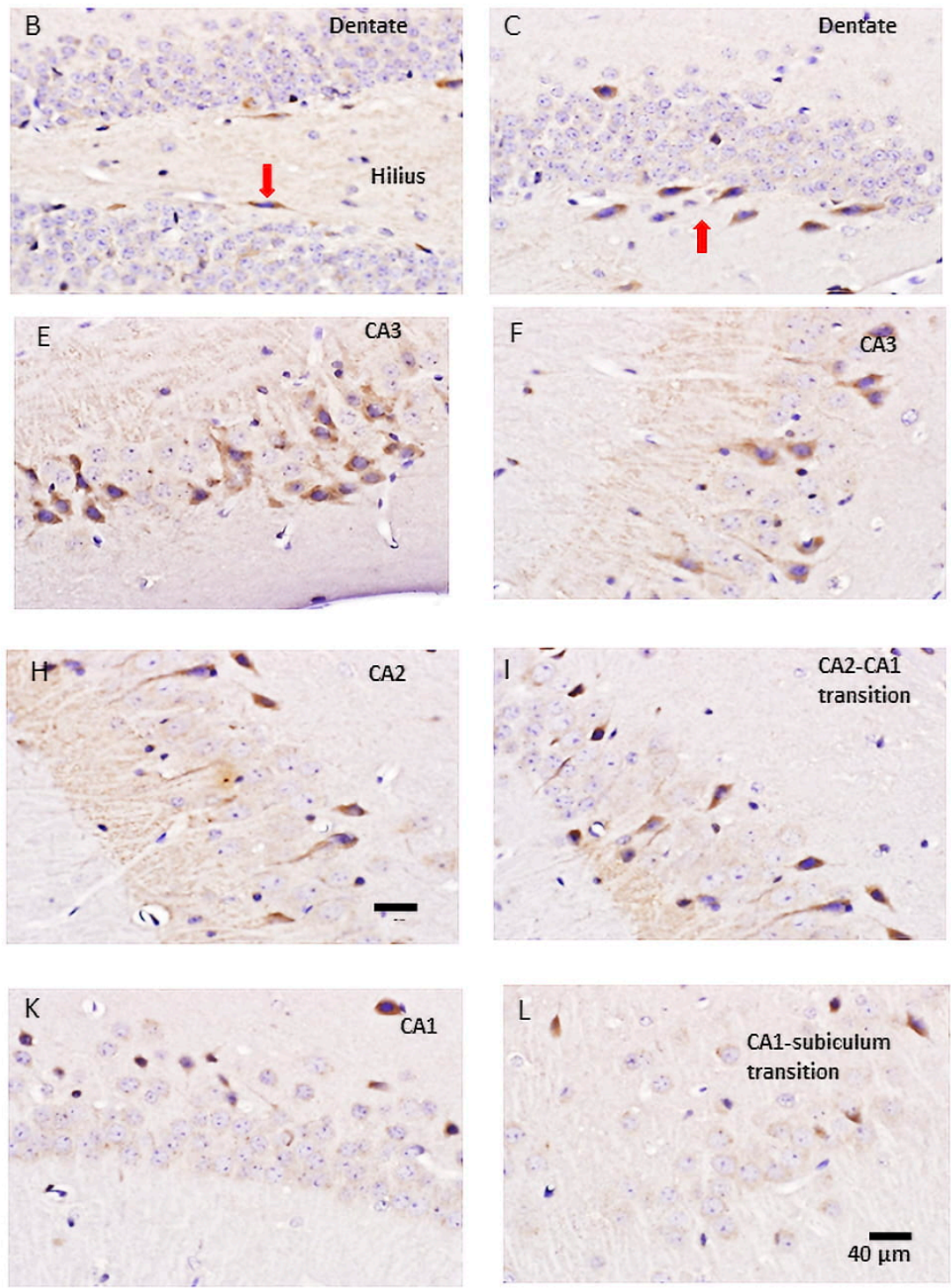

Fig. 4. Ab staining of cells in different regions of the hippocampus. (A) Shows the beginning of the DG in which most of the small round cells (i.e., granule cells) are unstained by the anti-PIEZO2 Ab. However, there was a cluster of stained cells at the crest/tip of the gyrus (red arrow). (B) Shows the adjacent region of the DG in which only 2-3 spindle-shaped cells (one indicated by red arrow) located in the subgranular zone show Ab staining. (C) A continuing region further along the DG showing 7 spindle-shaped cells stained by the anti-Piezo2 Ab. At least in this slice, the DG region only showed 16 out of 1149 cells with clear brown staining (i.e., 1.4\%). (D) Shows the hilus/C4 region with at least one darky-stained cell (red arrow) but only 2 out of the 72 neurons Ab stained. (E-G) In this slice, the CA3 region showed the highest density of Ab staining, with 50 out of 140 cells stained (36\%) and displayed unitary thick processes (i.e., apical dendrites) consistent with pyramidal neurons. (H) The CA2 region shows a somewhat lower density of stained neurons, with 15 out of 71 cells stained (22\%). (I) A region near the CA2-CA1 transition displays a further reduced density of stained neurons (23/305, i.e., $~ 8 \%)$. (J-K) CA1 regions showing even fewer stained neurons. (L) CA1 region transitioning into the subiculum that displayed no Ab-stained cells.

sagittal slices in 2 different mice indicated that for a total of 1912 Ab stained cells, 1277 (67\%) showed a pyramidal-like morphology, 193 (10\%) displayed multiple processes, and 442 (23\%) were round with no observable processes.

In the case of the hippocampus, the $\mathrm{Ab}$ also showed selective $\mathrm{Ab}$ staining of neurons in specific regions of the hippocampus. Fig. 3A shows at low magnification the different regions (i.e., dentate gyrus (DG), CA1-4), and Fig. 3B shows a more magnified CA3 region in which several neurons show clear brown staining, some of which appeared pyramidal shaped with a prominent process. Fig. 4 shows individual regions of the hippocampus from the same slice indicating a higher abundance of Ab stained cells in the CA3-2 regions compared with other regions (i.e., DG, hilus/CA4 and CA1 regions). What appeared most obvious for the DG (see Fig. 4A-C) was that the most abundant cells in this region, the granule cells, showed no $\mathrm{Ab}$ staining (i.e., the round blue cells). However, near the crest of the DG, there was a small cluster of stained cells (red arrow Fig. 4A) and spindle-shaped cells located in the subgranular layer (red arrows Fig. 4B, C). 
Table 1. Antigenic peptides used to generate anti-PIEZO2 antibodies and their staining of DRG and brain neuron.

\begin{tabular}{ccccc}
\hline Ab & Antigenic peptide & DRG & CNS & Ref/Antibody \\
\hline 1 & C-termini 2440-2628 & $90 \%$ & Yes & Current, custom \\
2 & N-termini 351-485 & $\leq 60 \%$ & $\mathrm{~N} / \mathrm{T}$ & [22], custom \\
3 & Not reported & $\mathrm{N} / \mathrm{T}$ & Yes & [8], ProSci, 26-438 \\
4 & C-termini 2446-2568 & $\mathrm{N} / \mathrm{T}$ & Yes & HPA, HPA015986 \\
5 & N-termini 845-864 & $>90 \%$ & Yes & [10], Prosci/8613 \\
6 & Internal 1092-1104 & $>90 \%$ & Yes & [10], Alomone/APC090 \\
\hline
\end{tabular}

These cells may reflect possibly pyramidal basket cells or even neural precursor cells. The CA3-2 regions (Fig. 4E-I) had a higher density of stained cells. Some cells had a classical pyramidal neuron appearance with a triangular-shaped cell body and a single prominent dendritic process. In transitioning from the CA2 to the CA1, there was a marked decrease in the number of stained cells. Similar staining patterns were seen in other slices from a different mouse with a lack of staining of dentate granule cells and the most abundant staining of pyramidal-like neurons in the CA3-CA2 regions. Cell counts made in these hippocampal regions from brains slices from two different mice indicated stained cells in DG represented only $\sim 2 \%$ of the cells counted (i.e., $50 / 2350$ ) with no evident staining of DG granule cells. Similarly, in the hilus/C4 regions, only $\sim 4 \%$ of neurons (i.e., 13/370) showed staining. In comparison, in the $\mathrm{CA} 3$ and $\mathrm{CA} 2$ regions, there were $\sim 33 \%$ (i.e., $105 / 315$ ) and $24 \%$ (i.e., $39 / 160$ ), respectively, but only $\sim 5 \%(15 / 305)$ in the CA1 region.

Our results for Piezo2 expression in mouse neocortex (NC) and hippocampus (HC) can be compared with PIEZO2 expression reported by the Human Protein Atlas (HPA) using a different $\mathrm{Ab}$ generated against a shorter $\mathrm{PIEZO} 2$ peptide (see Table 1, Ref. [8, 10, 22]). This Ab also stained neurons but not glial cells in the human NC and HC (see Supplementary Fig. 2A). In this case, the $\mathrm{Ab}$ results were orthogonally validated by $\mathrm{PIEZO} 2$ gene expression in human $\mathrm{NC}$ and $\mathrm{HC}$ tissues [24, 25], see Supplementary Fig. $\mathbf{2 B}^{2}$. On this basis, HPA has assigned this Ab result an "Approved" reliability score in the 2021 revision of their website (Dr. Borbala Katona, HPA Tissue Atlas Team, Uppsala University, personal communication). In another recent study of Piezo2 expression in rat peripheral and central neurons using two different anti-PIEZO2 Abs (Table 1), the Ab stained NC and HC neurons but not astrocytes [10]. Finally, a scRNA-seq study [26] has confirmed Piezo2 gene expression in mouse HC, with relatively higher gene expression in CA3 compared with CA2 and CA1 regions but with the DG cells showing a high transcript copy number ([26], and personal communication, Dr. Andres Vieira). It remains to be determined whether this reflects a real difference between gene transcription and translation of functional protein in hippocampal neurons.

\footnotetext{
2 https://www.proteinatlas.org/ENSG00000154864-PIEZO2/tissue
}

3.3 The Ab stains Purkinje cells but not granule cells in the cerebellar cortex

As indicated in Fig. 5, the Ab selectively stained Purkinje cells $(\mathrm{PC})$ in the cerebellar cortex, including their relatively large cell bodies and the branching dendrites that extend into the molecular cell layer (Fig. 5B). In marked contrast, the granule cells (GC) that form the granular layer showed no evidence of staining. This all-or-none selectivity in staining PC vs. GC again highlights the selectivity in Piezo2 expression given a PC:GC ratio of 1:3000 in the cerebellar cortex [27]. Interestingly, as indicated in Fig. 5A (i.e., yellow circle), some neurons embedded within the cerebellar white matter (arbor vitae) and possibly representing projection neurons within a deep cerebellar nucleus also showed Ab staining. Similar selective staining of $\mathrm{PC}$ in the human cerebellar cortex has been previously reported by the HPA using another anti-PIEZO $\mathrm{Ab}$ [24] (see Supplementary Fig. 2C and Table 1) . $^{3}$.

\subsection{The Ab selectively stains mitral cells in the olfactory bulb}

Fig. 6A shows a low magnified image of the mouse $\mathrm{OB}$ and indicates $\mathrm{Ab}$ staining of the $\mathrm{OB}$ neuropil with the circular glomeruli delineated by the hematoxylin stain. Fig. 6B$D$ show progressively magnified images of the same $O B$ indicating the strongest brown staining was of the mitral cells (MC) that form a distinct boundary cell layer separating the plexiform from the granular cell layers. The neuropil staining in the external plexiform layer presumably represents stained mitral cell dendrites that project to the glomeruli, where they receive synaptic inputs from primary olfactory neurons (PONs). Tufted cells located in the external plexiform layer, which also receive synapses from PONs, did not appear stained. In addition, as indicated in Fig. 6D, the most abundant cells of the OB, the granule cells, showed no evidence of positive (brown) staining.

This expression of Piezo2 in the MCs of the OB was unanticipated, especially given their well-recognized role in processing primary olfactory sensory inputs with no prior suspected intrinsic mechanosensitive function. However, a recent study using single nucleus RNA sequencing indicates that Piezo2 is a genetic marker of specific MCs within the mouse OB [28].

\section{Discussion}

The findings here that Piezo2 is expressed in select neurons of mouse brain augments earlier studies showing Piezo2 expression in isolated rodent brain tissues $[2,7,8]$. Furthermore, as described in each specific results section, the Piezo2 expression we observed in select mouse brain neurons has been confirmed by other independent proteomic and/or transcriptomic studies of rodent and/or human brain [10, 2426, 28] (and see Supplementary Fig. 2). The all-or-none, cell-type-specific Piezo2 expression in mouse brain neurons

\footnotetext{
3 https://www.proteinatlas.org/ENSG00000154864-PIEZO2/tissue/c erebellum
} 

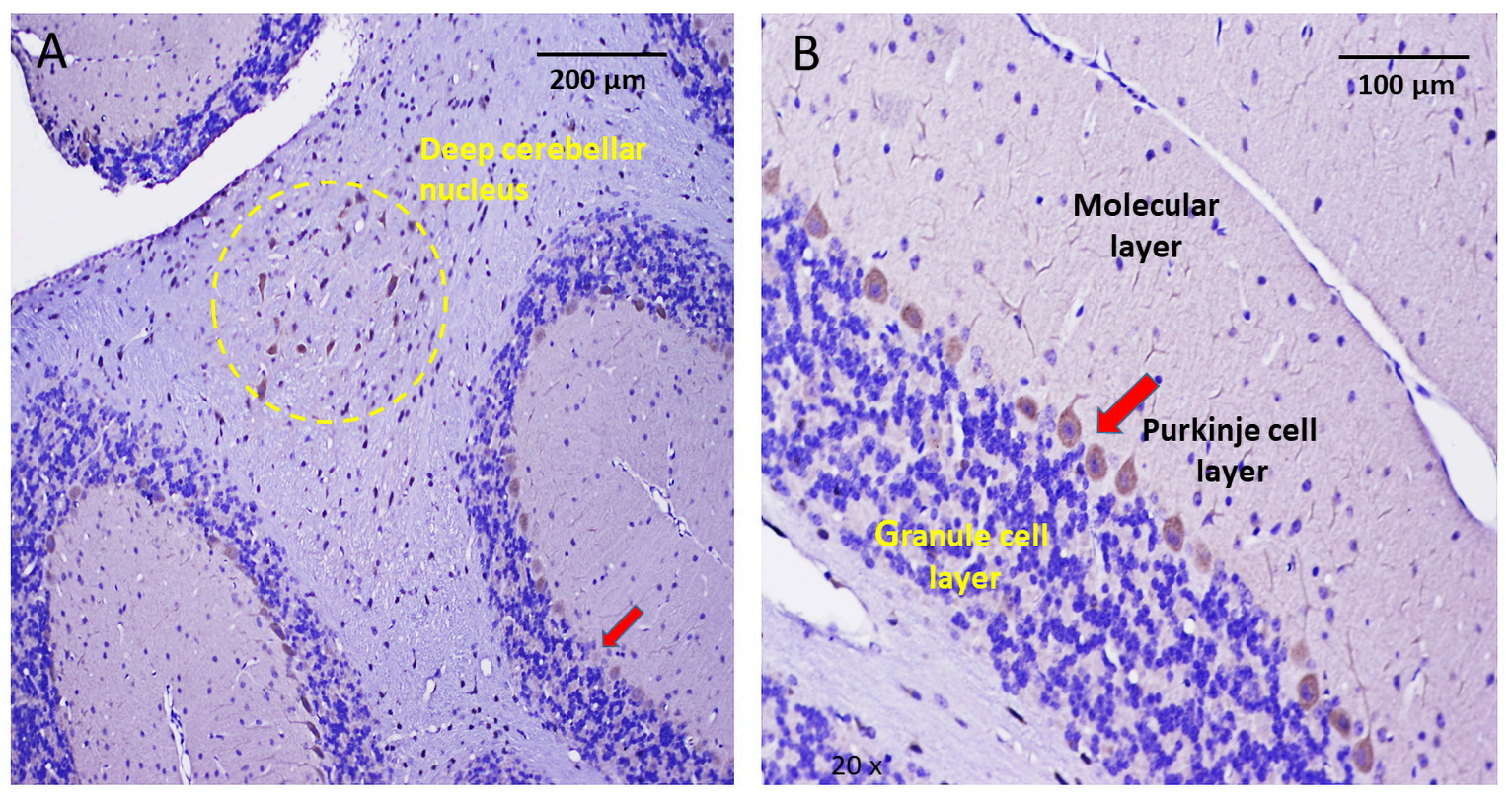

Fig. 5. Immunohistochemical localization of Piezo2 in the cerebellum. (A) Low magnification of a cerebellar slice indicating the stained Purkinje cell layer (red arrow) that separates the granule and molecular cell layers. The yellow dashed circle within cerebellar white matter (arbor vitae) encloses a group of stained neurons, presumably part of a deep cerebellar nucleus. (B) Higher magnification of the cerebellar cortex showing the characteristic single layer of Purkinje cells, which appear uniformly stained by the anti-PIEZO2 Ab. In contrast to the stained Purkinje cells, the more numerous granular cells within the granular cell layer were not stained. Similarly, apart from Purkinje cell dendrites, there was no pronounced Ab staining of cells within the molecular layer.

is also interesting in relation to the stochastic on-off transcriptional switch that seems to regulate Piezo1 expression in mouse and human brain [16]. In this case, pathological conditions, including Alzheimer's disease (AD) and infection, can switch Piezo1 expression between neurons and astrocytes [16-18].

In contrast to the increasing proteomic and transcriptomic evidence for Piezo2 brain expression in brain neurons, there is no direct evidence for functional expression. However, a recent patch-clamp study of mouse brain slices has reported single "Piezo-like" pressure-activated single-channel currents in neocortical and hippocampal pyramidal neurons [9] and another study, using $\mathrm{Ca}^{2+}$ imaging and atomic force microscopy, indicated pressure-activated $\mathrm{Ca}^{2+}$ currents in isolated neocortical and hippocampal neurons, possibly reflecting Piezo channels but also other putative mechanosensitive channels [29]. Therefore, although confirmatory evidence for Piezo2 channel involvement is still required, the simple recording of pressure-activated currents in brain neurons makes it worthwhile to consider the possible pressure changes in vivo that could activate these channels in brain neurons. Already the finding that rats exposed to large exogenous pressures pulses, simulating blast forces, show altered Piezo2 channel expression in neocortical and hippocampal tissues $[7,8]$ at least suggests that the abnormal activation of Piezo 2 may contribute to the acute and often-reversible deficits in memory, consciousness and motor-sensory functions associated with concussion and mild TBI. This idea would at least be in-line with patch-clamp results that re- ported even brief $(\sim 1 \mathrm{~s})$ pressure pulses of $\geq 25 \mathrm{mmHg}$ applied to the patch could activate channels that triggered high frequency $(30 \mathrm{~Hz})$ spiking in neocortical and hippocampal neurons [9].

Interestingly, TBI is only one of several neuropathological conditions, including hydrocephalus, cerebral hemorrhage and brain tumors, known to elevate the normally low $(<10$ $\mathrm{mm} \mathrm{Hg}$ ) baseline intracranial pressure (ICP) to higher levels ( $>25 \mathrm{mmHg}$ ), all with serious clinical consequences [30-33]. Given that it has been directly demonstrated that pressure pulses as low as $\leq 5 \mathrm{mmHg}$ can activate Piezo2 channels [34], it would not be surprising that pressures 5-fold higher would induce abnormal Piezo2 activities and contribute to deficits in brain function. A special case is normal pressure hydrocephalus (NPH), in which enlarging ventricles compress the brain parenchyma [35]. Interestingly, the dementia associated with the NPH is often misdiagnosed as Alzheimer's disease but can be distinguished by ventriculoperitoneal shunts that reduce ICP and often rapidly reverse this form of dementia [35].

Although expression of Piezo2 in brain neurons may confer no survival advantage, the apparent Piezo2 related response to concussion/TBI (i.e., with ICP $>25 \mathrm{mmHg}$ ) would seem a disadvantage. In this case, Piezo2 expression might only be maintained if it did confer an additional selective advantage, possibly involving improved normal brain performance. Therefore, a more relevant and interesting question is whether smaller changes in ICP $(\leq 10 \mathrm{mmHg})$ that occur in the healthy brain might also produce changes in channel 

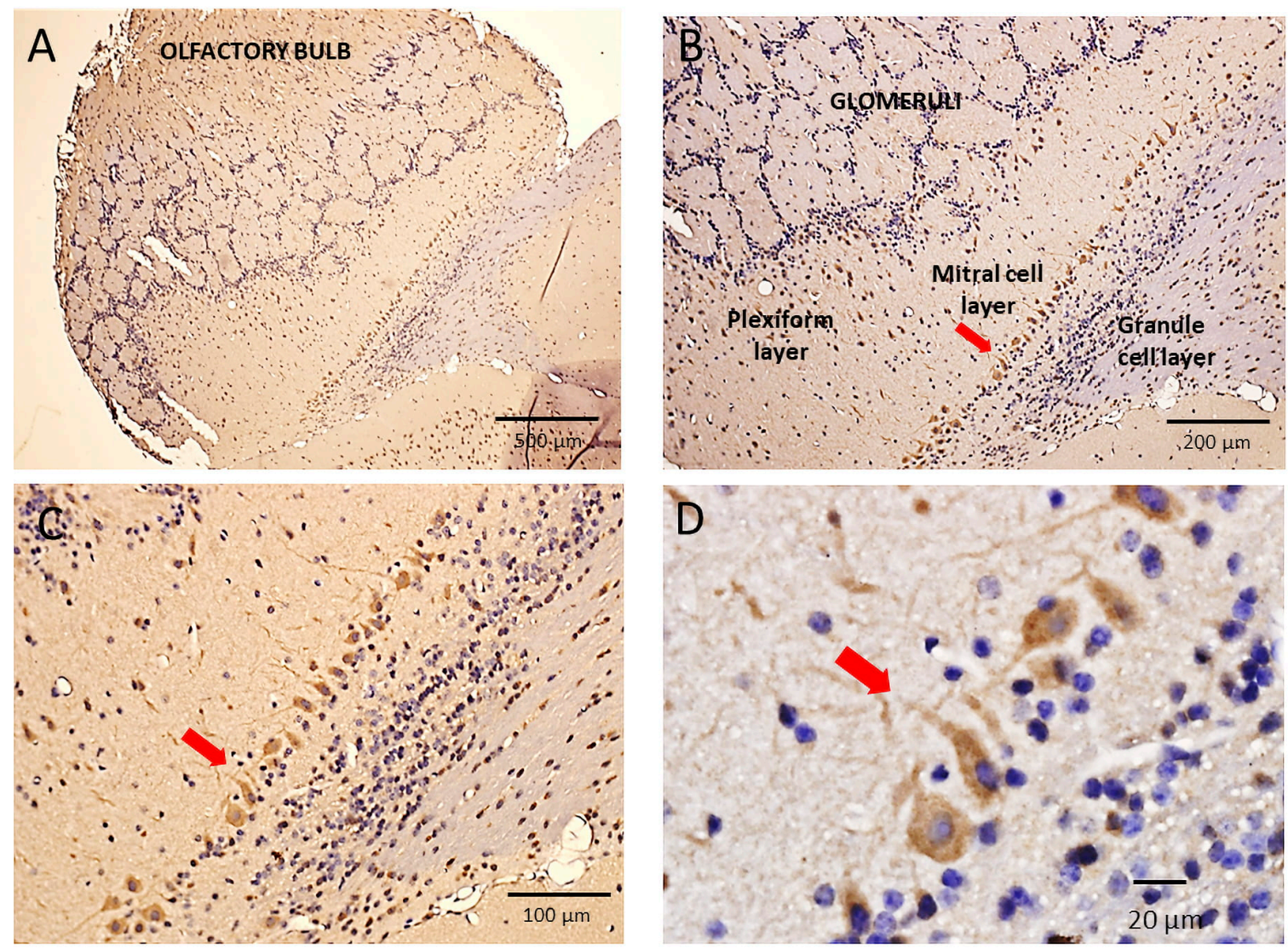

Fig. 6. Immunohistochemical localization of Piezo2 in the mouse olfactory bulb. (A) Shows a low magnification image of the mouse olfactory bulb (OB) with its characteristic circular/spherical glomeruli structures spanning the OB. These glomeruli include the synaptic connections formed between primary olfactory nerve (PON) axons and mitral cell dendrites. (B-C) Higher magnification images (10× and $20 \times$ objectives) of the same slice showing the uniformly stained layer of mitral cell bodies (red arrows) that separate the external plexiform layer from the internal plexiform and granule cell layers. The mitral cells represent the primary projection neuron of the $\mathrm{OB}$ and project their axons to the piriform and entorhinal cortices and the amygdala. The external plexiform layer includes the primary and lateral dendrites of the mitral cells that extend into and throughout the plexiform layer to reach the glomeruli. Also within this layer are the cell bodies and dendrites of the tufted cells, which did not appear stained. (D) A still higher magnified image $(60 \times$ objective) showing the morphology and staining of the mitral cells bodies and dendrites and the absence of staining of granule cells and the tufted cells in the granule cell and external plexiform layers, respectively.

activity and thereby somehow enhance normal brain function. In normal healthy subjects, ICP registers at typically low baseline values ( $\leq 10 \mathrm{mmHg}$ ). However, ICP also undergoes metronomic-like, pulsatile changes (typically $\leq 5 \mathrm{mmHg}$ ) involving rapid ( $200 \mathrm{~ms})$ pulses with each heartbeat [3033], as well as much slower (5-10 s) pulses associated with breathing [36-38]. Moreover, specific volitional breathing practices-involving slow inspiration/expiration cycles and/or diaphragmatic vs. thoracic breathing-performed to improve attention or reduce stress/anxiety cause even larger pulsatile changes in ICP [38]. This raises the intriguing possibility that the presence of pressure-activated channels allows specific neurons to transduce these pulsatile ICP changes, thereby entraining the rhythmic neural network oscillations measured with EEG and shown to underlie specific behavioral states associated with mindful breathing [39-41]. In this case, pulsatile fluctuations in ICP may have started as epiphenomena [1], representing the unavoidable cerebral responses to breathing and cardiac rhythms. However, over time selective pressure favored Piezo2 expression and its ICP pulse transduction because it introduced a new mechanism for synchronizing neural network communication, especially within the much larger brains of humans.

Direct experimental evidence for the actual idea that breathing can induce entrainment of neural networks goes back 80 years, with the discovery by Edgar Adrian that nasal breathing in rodents causes the rhythmic firing of mitral neurons within the olfactory bulb and neurons within neurons the piriform cortex [42]. Many subsequent studies have extended his discovery by showing that nasal breathing not only entrains $\mathrm{OB}$ oscillations at the rodent's breathing frequency (0.5-5 Hz) but also modulates the amplitude of higher frequency oscillations $(80-120 \mathrm{~Hz})$ in the OB as well as other downstream brain regions including the hippocampus and neocortex [43-45]. Significantly, these neural network oscillations have also been associated with specific changes in ro- 


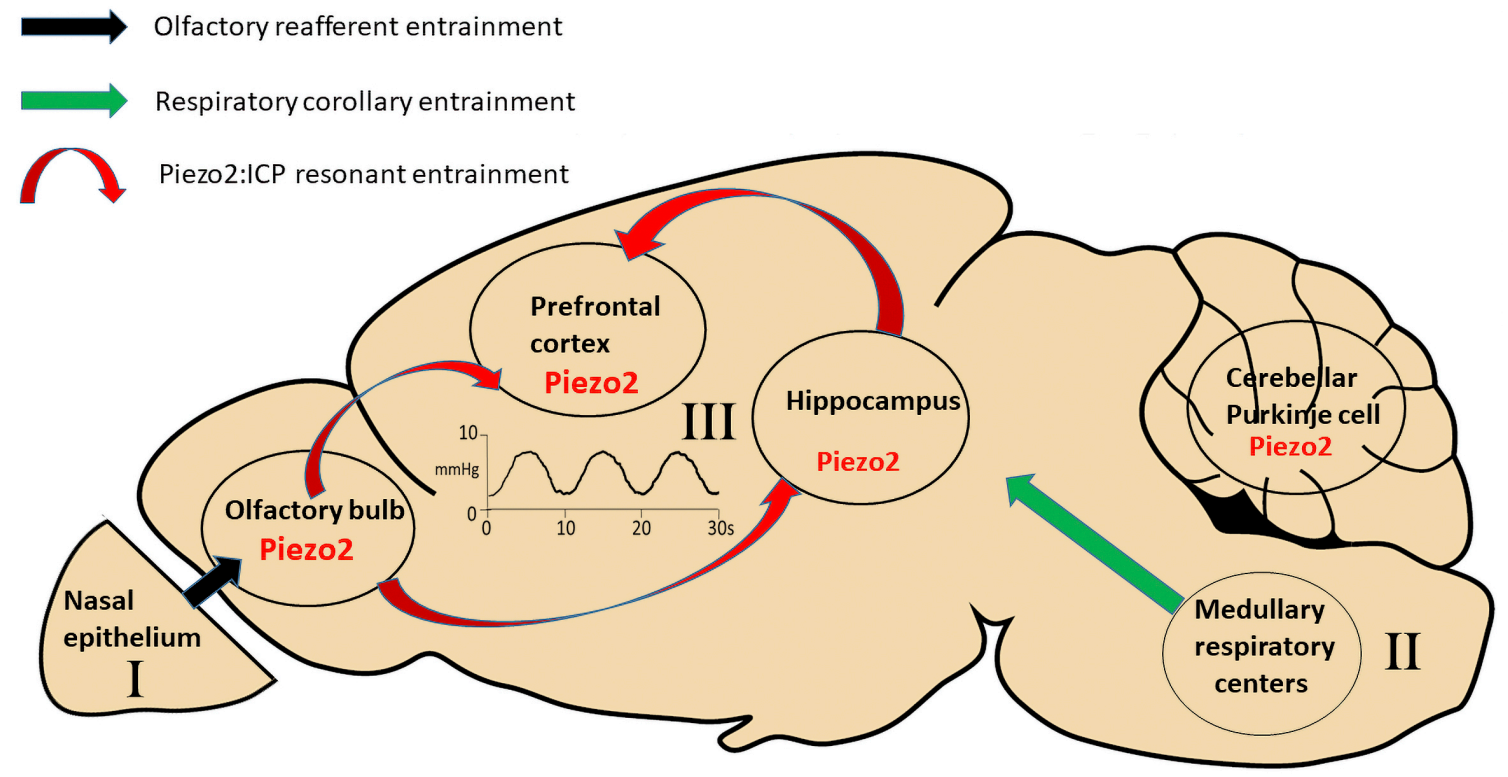

Fig. 7. Different mechanisms for respiration-induced entrainment of neural networks in widely spaced regions of the brain. The first mechanism involves respiratory olfactory re-afferent discharge (ORD). It originates in the nasal epithelium, where primary olfactory neurons (PONs), which express olfactory G protein-coupled receptors (GPCRs) that are also mechanosensitive, can detect the nasal airflow pressure changes associated with nasal breathing. The PONs, through their direct synaptic connections (black arrow) with mitral cells and tufted cells in the OB, cause respiratory entrainment of the neural activity within the OB. In turn, the OB entrains neural activity in the hippocampus and prefrontal cortex through their monosynaptic and/or polysynaptic connections (not shown) with these brain regions. The second mechanism depends upon respiratory corollary discharge (RCD) arising from the neurons in the medullary respiratory nuclei that control the diaphragm muscles and send efferent copy discharges to high brain regions (e.g., hippocampus and neocortex) via yet to be identified synaptic pathways. The third mechanism, intrinsic resonance discharge (IRD), depends upon the pressure-sensitive channel, Piezo2, expressed in mitral cells of the $\mathrm{OB}$ and pyramidal cells of the hippocampus and neocortex. Piezo2, based on its demonstrated high sensitivity to low-pressure pulses $(\leq 5 \mathrm{mmHg}$, is proposed to transduce the ICP pulse associated with the respiratory cycle, thereby providing a synchronizing clock for brain activity. Finally, Piezo2 expression shown in cerebellar Purkinje cells may explain the Purkinje cell's sniffing-dependent activation in the human cerebellum.

dent behaviors, including whisking, memory formation and emotional (fear) responses [46-55]. Moreover, the many observations related to respiration-locked oscillations seen in rodents have now been confirmed in humans, using either intracranial EEG recording from epileptic patients or highdensity EEG recording from healthy subjects [40, 41, 56, 57].

At least three different, non-mutually exclusive mechanisms may explain respiration-induced neural network oscillations [43-46]. The first, called olfactory re-afferent discharge (ORD), proposes that nasal airflow during nasal breathing activates mechanosensitive PON that, in turn, activate via their direct synaptic connections, MCs within the $\mathrm{OB}$ [58] (see Fig. 7). In this mechanism, the OB acts as a "global clock" for other brain regions, synchronizing via its synaptic connections, neural networks across widespread brain regions, including the hippocampus and neocortex [44, 45]. Evidence supporting this mechanism is that tracheotomy or ablation of the nasal epithelium (or removal of the OB) in rodents reduces the synchronized activity in the $\mathrm{OB}$ and/or downstream brain regions [42, 43, 59, 60]. Moreover, in both humans and rodents, air pressure pulses delivered to the nostrils can restore and/or alter the oscillation frequency $[51,57]$.
The second mechanism referred to as "respiratory corollary discharge" (RCD) depends upon efferent copy discharges from neurons in the brain stem nuclei that regulate breathing [61-63] (see also Fig. 7). Support for this mechanism includes the finding that although tracheotomy or ablation of the nasal epithelium reduces the neural oscillations in rodents, they do not completely block them $[46,63]$. Moreover, in humans, the oscillations persist during mouth breathing and, therefore, in the absence of any nasal airflow [56, 63]. Further evidence for the RCD mechanism is that neurons in the brain stem respiratory nuclei form connections with neurons in the locus coeruleus that via their projections may alter activity in widely spaced neural networks throughout the brain [62]. An added twist to both the RCD and ORD mechanisms is the recent report [64] that low frequency (i.e., $\theta=3.5-7.5 \mathrm{~Hz}$ ) activity in neural circuits may feedback in a top-down manner on the brain stem nuclei to influence respiration frequency. Changes in respiratory frequency then, in turn, feedforward (i.e., bottom-up) on neural circuits to increase the power of higher-frequency oscillations (i.e., slow $\gamma=25-50 \mathrm{~Hz}$ ) [64].

The third mechanism and the least investigated, we refer to as intrinsic resonance discharge (IRD). In this case, the intrinsic properties of the neural networks involving either intrinsic neuronal membrane properties or synaptic micro- 
circuitry dictate that they fire or resonate at a frequency close to the respiratory frequency [41]. The evidence for this mechanism is again that respiration-entrained oscillations are retained during mouth breathing or in the absence of nasal airflow or functional PONs [46, 56, 63]. Moreover, IRD may result in part account for a somewhat puzzling feature of the ORD mechanism, which relates to the mechanosensitivity of the PON and their odor-sensitive G-protein coupled receptors (GPCRs) [58]. This GPCR mechanosensitivity depends upon a second messenger pathway and consequently displays long latencies $(-300 \mathrm{~ms})$ in response to pressure pulses [58].

Furthermore, because of GPCR adaptation, their maximum frequency response to repetitive pressure pulse stimuli is only $\sim 0.5 \mathrm{~Hz}$ [58]. This is significantly lower than the rodent's range of breathing frequency $(1-10 \mathrm{~Hz}$ but as high as $15 \mathrm{~Hz}$ during sniffing) as well as the entrainment frequency induced in the MCs of the OB $(1-10 \mathrm{~Hz})$ [65]. Although the convergence of many PON inputs to the MCs may still generate rhythmic activity that follows respiration, even when only a fraction of the PONs responding with each respiratory cycle [58], an alternative or reinforcing mechanism could be that the MCs themselves possess an intrinsic mechanosensitive resonance to the breathing cycle. Specifically, we hypothesize that Piezo2 in MCs and neurons in the neocortex and hippocampus confer an intrinsic resonance that reinforces their entrainment by the breathing rhythm via their transduction pulsatile ICP changes (Fig. 7). Because stretchactivated channels, including Piezo channels, are characterized by their fast gating [66] and ability to accurately track relative high frequency (e.g., $\geq 5 \mathrm{~Hz}$ ) pressure pulses $[67,68]$, they could confer resonance for breathing-associated, as well as the even higher frequency cardiac-associated, ICP pulses [30-32, 69-71]. Moreover, the expression of Piezo2 in cerebellar PC may also explain the sniffing-dependent activation of the human cerebellum [72] and the highly correlated slow $(\sim 1 \mathrm{~Hz})$ oscillations seen between mouse PCs [73]. An intrinsic mechanism could also allow for top-down modulation of synchronization [64] by steady-state pressure and/or membrane potential switching of the pressure sensitive channelgating mode [67].

An intrinsic Piezo2/ICP resonance mechanism may also have acquired a more predominant role for human breathing-induced entrainment because of additional selective pressures. In particular, in humans, nasal breathing is not obligatory as in rodents. In addition, olfaction has become a relatively unimportant sensory function, adding little advantage for survival, compared with rodents where olfaction, sniffing and whisking are tightly linked and critical for their normal behavior and survival. Moreover, humans appear to be the only species capable of volitionally switching breathing patterns (e.g., forced inspiration/expiration cycles) to promote mood, cognition, and emotional changes. Humans may also be unique in that they can choose to modulate volitionally through their breathing, both their heartbeat and emo- tions $[69,70]$. Interestingly, a study of single-unit recording from epileptic patients indicated a higher proportion of hippocampal and amygdala neurons show entrainment with the cardiac cycle compared with the respiratory cycle [71].

Regardless of the exact mechanism, a key issue for all mechanisms is their reliability and degree of synchronization that they can induce within widely separated neural networks. This synchronization has special implications for the unity of experience related to perception, motor control, and memory retrieval, especially in the larger human brain. In the case of the first two mechanisms, the overall synchronization will depend upon signaling delays within multisynaptic, axonal transmission pathways. In comparison, a Piezo2/ICP pulse dependent IRD mechanism will only be rate-limited by the speed of ICP pulse transmission throughout the brain, powered by the perennial actions of the respiratory and cardiac pumps. In the ideal case, when Pascal's law applies, namely a rigid, fluid-filled and closed container, a pressure pulse will spread throughout the container almost instantaneously at the speed of sound (i.e., $\sim 1500 \mathrm{~m} / \mathrm{s}$ ) [31]. However, this may represent an upper estimate since the brain contained within the skull is a semi-closed system that involves cerebral venous outflow and arterial inflow [3032]. Moreover, CSF and blood circulate between the brain and spinal cord, particularly during inspiration and expiration [36-38]. In addition, the brain parenchyma, ventricles, and vasculature are themselves compliant rather than rigid, which will also tend to dissipate and slow the spread of ICP changes.

In one notable study [74], aimed at measuring ICP transmission in the human brain, two ICP sensors, placed $5 \mathrm{~cm}$ apart in the lateral ventricle and brain parenchyma measured a phase shift of $10 \mathrm{~ms}$ [74]. This would predict a pulse velocity of only $5 \mathrm{~m} / \mathrm{s}$. (i.e., which compares with a predicted $50 \mu \mathrm{s}$ phase shift for a velocity of $1500 \mathrm{~m} / \mathrm{s}$ ). However, there are two caveats regarding this lower estimate. First, the ICP pulse does not have a single origin but arises throughout the arterial tree [30]. Second, the pressure pulsations may occur within the dense microvascular embedded in the parenchyma so that no individual neuron is more than $100 \mu \mathrm{m}$ from a pulsating vessel [75]. Both effects would minimize delays and thereby synchronize the ICP pulse transmission to all neurons within neural networks throughout the brain.

\section{Conclusions}

The current immunohistochemical evidence for Piezo2 expression in specific mouse brain neurons is now supported by independent rodent and human brain studies using different anti-PIEZO2 Abs generated against different regions of the PIEZO2 protein (Table 1). In specific cases, the Abs have met additional validation criteria ${ }^{4}$ including orthogonal (i.e., IHC/ transcriptomic data correspondence) [24-26, 28] (see

\footnotetext{
4 https://www.proteinatlas.org/about/antibody+validation
} 
Supplementary Figs. 1,2) and genetic criteria [10]. Nevertheless, and ideally, verification of the IHC results with $\mathrm{Ab}$-independent techniques is still required (i.e., using GFPtagged Piezo2 transgenic mice [76], GENSAT). Furthermore, since Piezo1 shows expression in rodent and human brain neurons [16-18] and both Piezo1 and Piezo2 function in peripheral baroreception [5], it will be important to establish their individual expression patterns and roles in specific brain neurons. However, the biggest future challenge will be demonstrating that Piezo channel activity participates in the entrainment of neural network oscillations in living, breathing animals. One approach may involve using conditional Piezo knockout mice to compare respiratory entrained neural network oscillations with those reported previously for wild-type mice [43-49]. It should also be interesting to study human patients that show specific loss of PIEZO2 function [77] and compare how their measured EEG and behavioral responses to specific breathing protocols differ from those seen in normal human subjects [39-41, 56, 57].

Finally, recalling the fuller and particularly insightful quote from Hutcheon and Yarom [1]:

"A question therefore arises: are resonances used by neurons, or are they simply epiphenomena? A broad answer to this question is that, in nature, epiphenomena seldom remain epiphenomena for long; they are the raw material for evolutionary advances. It would be surprising to find that the brain has not found a use for a set of mechanisms capable of tuning neurons to specific frequencies, particularly in light of the prevalence of robust brain rhythms."

Here, Hutcheon and Yarom are referring to, at the time, the relatively newly discovered roles of the various EEG recorded electrical brain rhythms [78]. However, it may also turn out the metronomic-like and perennial ICP pulses, transduced by pressure-sensitive channels, prove even more pivotal in tuning and synchronizing the brain to the fundamental rhythms of life [44, 45].

\section{Abbreviations}

$\mathrm{Ab}$, antibody; $\mathrm{AD}$, Alzheimer's disease; CSF, cerebrospinal fluid; DG, dentate gyrus; DRG, dorsal root ganglion; EEG, electroencephalography; GFP, green fluorescent protein; GC, granule cell; GPCR, G-protein coupled receptor; HC, hippocampus; HPA, Human Protein Atlas; ICP, intracranial pressure; IHC, immunohistochemistry; IRD, intrinsic resonance discharge. MC, mitral cell; NC, neocortex; NPH, Normal Pressure Hydrocephalus; OB, olfactory bulb; ORD, olfactory re-afferent discharge; PC, Purkinje cell; PON. primary olfactory neuron; PrEST, protein epitope signature tag; RCD, respiratory corollary discharge; snRNA-seq, single nucleus RNA sequencing; scRNA-seq, single cell RNA sequencing; TBI, traumatic brain injury.

\section{Author contributions}

JW surgically isolated and prepared the mouse brains and DRG for slicing. OH conceived the study, analyzed and inter- preted the IHC experiments and wrote the manuscript. All authors read and approved the final manuscript.

\section{Ethics approval and consent to participate}

Mice were housed in the animal facility at UTMB, Galveston and all experimental protocols were approved by the Animal Care and Use Committee at the UTMB (The ethical approval number for our animal protocol is 0907049) and are in accordance with the NIH Guide for the Care and Use of Laboratory Animals.

\section{Acknowledgment}

We thank Jin Mo Chung for providing his laboratory resources and support for Jigong Wang. We also thank Thomas Green for critical reading the manuscript, and Daniel Jupiter, and Andrzej Kudlicki for useful discussions. We also acknowledge the technical assistance of Kerry Graves of the UTMB Pathology core facility for preparing the tissues for IHC and Kenneth Escobar in providing the microscopy facilities.

\section{Funding}

The project was supported by a UTMB bridging grant to OPH and an NIH Grant NS03168 to Jin Mo Chung.

\section{Conflict of interest}

The authors declare no conflict of interest.

\section{Supplementary material}

Supplementary material associated with this article can be found, in the online version, at https://www.imrpress.com/j ournal/JIN/20/4/10.31083/j.jin2004085.

\section{References}

[1] Hutcheon B, Yarom Y. Resonance, oscillation and the intrinsic frequency preferences of neurons. Trends in Neurosciences. 2000; 23: 216-222.

[2] Coste B, Mathur J, Schmidt M, Earley TJ, Ranade S, Petrus MJ, et al. Piezo1 and Piezo2 are essential components of distinct mechanically activated cation channels. Science. 2010; 330: 55-60.

[3] Kefauver JM, Ward AB, Patapoutian A. Discoveries in structure and physiology of mechanically activated ion channels. Nature. 2020; 587: 567-576.

[4] Szczot M, Nickolls AR, Lam RM, Chesler AT. The Form and Function of PIEZO2. Annual Review of Biochemistry. 2021; 90: 507-534.

[5] Zeng WZ, Marshall KL, Min S, Daou I, Chapleau MW, Abboud FM, et al. PIEZOs mediate neuronal sensing of blood pressure and the baroreceptor reflex. Science. 2018; 362: 464-467.

[6] Nonomura K, Woo SH, Chang RB, Gillich A, Qiu Z, Francisco AG, et al. Piezo2 senses airway stretch and mediates lung inflationinduced apnoea. Nature. 2017; 541: 176-181.

[7] Sajja VSSS, Ereifej ES, VandeVord PJ. Hippocampal vulnerability and subacute response following varied blast magnitudes. Neuroscience Letters. 2014; 570: 33-37.

[8] Heyburn L, Abutarboush R, Goodrich S, Urioste R, Batuure A, Statz J, et al. Repeated Low-Level Blast Overpressure Leads to Endovascular Disruption and Alterations in TDP-43 and Piezo2 in a Rat Model of Blast TBI. Frontiers in Neurology. 2019; 10: 766. 
[9] Nikolaev YA, Dosen PJ, Laver DR, van Helden DF, Hamill OP. Single mechanically-gated cation channel currents can trigger action potentials in neocortical and hippocampal pyramidal neurons. Brain Research. 2015; 1608: 1-13.

[10] Shin SM, Moehring F, Itson-Zoske B, Fan F, Stucky CL, Hogan $\mathrm{QH}$, et al. Piezo2 mechanosensitive ion channel is located to sensory neurons and nonneuronal cells in rat peripheral sensory pathway: implications in pain. Pain. 2021; 162: 2750-2768.

[11] Taberner FJ, Prato V, Schaefer I, Schrenk-Siemens K, Heppenstall PA, Lechner SG. Structure-guided examination of the mechanogating mechanism of PIEZO2. Proceedings of the $\mathrm{Na}-$ tional Academy of Sciences. 2019; 116: 14260-14269.

[12] Wang L, Zhou H, Zhang M, Liu W, Deng T, Zhao Q, et al. Structure and mechanogating of the mammalian tactile channel PIEZO2. Nature. 2019; 573: 225-229.

[13] van der Loos CM. Multiple immunoenzyme staining: methods and visualizations for the observation with spectral imaging. The Journal of Histochemistry and Cytochemistry. 2007; 56: 313-328.

[14] Wang J, La JH, Hamill OP. PIEZO1 is Selectively Expressed in Small Diameter Mouse DRG Neurons Distinct from Neurons Strongly Expressing TRPV1. Frontiers in Molecular Neuroscience. 2019; 12: 178.

[15] Roh J, Hwang SM, Lee SH, Lee K, Kim YH, Park CK. Functional Expression of Piezo1 in Dorsal Root Ganglion (DRG) Neurons. International Journal of Molecular Science. 2020; 21: 3834.

[16] Satoh K, Hata M, Takahara S, Tsuzaki H, Yokota H, Akatsu H, et al. A novel membrane protein, encoded by the gene covering KIAA0233, is transcriptionally induced in senile plaque-associated astrocytes. Brain Research. 2006; 1108: 19-27.

[17] Blumenthal NR, Hermanson O, Heimrich B, Shastri VP. Stochastic nanoroughness modulates neuron-astrocyte interactions and function via mechanosensing cation channels. Proceedings of the National Academy of Sciences of the United States of America. 2014; 111: 16124-16129.

[18] Hall CM, Moeendarbary E, Sheridan GK. Mechanobiology of the brain in ageing and Alzheimer's disease. European Journal of Neuroscience. 2021; 53: 3851-3878.

[19] Zhang M, Wang Y, Geng J, Zhou S, Xiao B. Mechanically Activated Piezo Channels Mediate Touch and Suppress Acute Mechanical Pain Response in Mice. Cell Reports. 2019; 26: 14191431.e4.

[20] Usoskin D, Furlan A, Islam S, Abdo H, Lönnerberg P, Lou D, et al. Unbiased classification of sensory neuron types by large-scale single-cell RNA sequencing. Nature Neuroscience. 2015; 18: 145153.

[21] Sharma N, Flaherty K, Lezgiyeva K, Wagner DE, Klein AM, Ginty DD. The emergence of transcriptional identity in somatosensory neurons. Nature. 2020; 577: 392-398.

[22] Ranade SS, Woo SH, Dubin AE, Moshourab RA, Wetzel C, Petrus $\mathrm{M}$, et al. Piezo2 is the major transducer of mechanical forces for touch sensation in mice. Nature. 2014; 516: 121-125.

[23] Ma S, Cahalan S, LaMonte G, Grubaugh ND, Zeng W, Murthy SE, et al. Common PIEZO1 Allele in African Populations Causes RBC Dehydration and Attenuates Plasmodium Infection. Cell. 2018; 173: 443-455.e12.

[24] Uhlén M, Fagerberg L, Hallström BM, Lindskog C, Oksvold P, Mardinoglu A, et al. Tissue-based map of the human proteome. Science. 2015; 347: 1260419.

[25] Ardlie KG, Deluca DS, Segrè AV, Sullivan TJ, Young TR, Gelfand ET, et al. The Genotype-Tissue Expression (GTEx) pilot analysis: multi-tissue gene regulation in humans. Science. 2015; 348: 648660.

[26] Machado JP, Athie MC, Matos AH, Lopes-Cendes I, Vieira AS. The transcriptome of rat hippocampal subfields. bioRxiv. 2021. (in press)

[27] Lange W. Cell number and cell density in the cerebellar cortex of man and some other mammals. Cell and Tissue Research. 1975; 157: 115-124.

[28] Zeppilli S, Ackels T, Attey R, Klimpert N, Ritola KD, Boeing S, et al. Molecular characterization of projection neuron subtypes in the mouse olfactory bulb. eLife. 2021; 10: e65445.

[29] Gaub BM, Kasuba KC, Mace E, Strittmatter T, Laskowski PR, Geissler SA, et al. Neurons differentiate magnitude and location of mechanical stimuli. Proceedings of the National Academy of Sciences. 2020; 117: 848-856.

[30] Czosnyka M, Pickard J. Monitoring and interpretation of intracranial pressure. Journal of Neurology, Neurosurgery and Psychiatry. 2004; 75: 813-821.

[31] Wagshul ME, Eide PK, Madsen JR. The pulsating brain: a review of experimental and clinical studies of intracranial pulsatility. Fluids and Barriers of the CNS. 2011; 8: 5 .

[32] Greitz D, Wirestam R, Franck A, Nordell B, Thomsen C, Ståhlberg F. Pulsatile brain movement and associated hydrodynamics studied by magnetic resonance phase imaging. the MonroKellie doctrine revisited. Neuroradiology. 1992; 34: 370-380.

[33] Andresen M, Hadi A, Petersen LG, Juhler M. Effect of postural changes on ICP in healthy and ill subjects. Acta Neurochirurgica. 2015; 157: 109-113.

[34] Shin KC, Park HJ, Kim JG, Lee IH, Cho H, Park C, et al. The Piezo2 ion channel is mechanically activated by low-threshold positive pressure. Scientific Reports. 2019; 9: 6446.

[35] Griffa A, Van De Ville D, Herrmann FR, Allali G. Neural circuits of idiopathic Normal Pressure Hydrocephalus: a perspective review of brain connectivity and symptoms meta-analysis. Neuroscience and Biobehavioral Reviews. 2020; 112: 452-471.

[36] Dreha-Kulaczewski S, Joseph AA, Merboldt KD, Ludwig HC, Gärtner J, Frahm J. Inspiration is the major regulator of human CSF flow. The Journal of Neuroscience. 2015; 35: 2485-2491.

[37] Dreha-Kulaczewski S, Joseph AA, Merboldt KD, Ludwig HC, Gärtner J, Frahm J. Identification of the Upward Movement of Human CSF In Vivo and its Relation to the Brain Venous System. Journal of Neuroscience. 2017; 37: 2395-2402.

[38] Aktas G, Kollmeier JM, Joseph AA, Merboldt KD, Ludwig HC, Gärtner J, et al. Spinal CSF flow in response to forced thoracic and abdominal respiration. Fluids and Barriers of the CNS. 2019; 16: 10.

[39] Moore A, Gruber T, Derose J, Malinowski P. Regular, brief mindfulness meditation practice improves electrophysiological markers of attentional control. Frontiers in Human Neuroscience. 2012; 6: 18.

[40] Zelano C, Jiang H, Zhou G, Arora N, Schuele S, Rosenow J, et al. Nasal Respiration Entrains Human Limbic Oscillations and Modulates Cognitive Function. The Journal of Neuroscience. 2016; 36: 12448-12467.

[41] Herrero JL, Khuvis S, Yeagle E, Cerf M, Mehta AD. Breathing above the brain stem: volitional control and attentional modulation in humans. Journal of Neurophysiology. 2018; 119: 145-159.

[42] Adrian ED. Olfactory reactions in the brain of the hedgehog. The Journal of Physiology. 1942; 100: 459-473.

[43] Fontanini A, Bower JM. Slow-waves in the olfactory system: an olfactory perspective on cortical rhythms. Trends in Neurosciences. 2006; 29: 429-437.

[44] Heck DH, McAfee SS, Liu Y, Babajani-Feremi A, Rezaie R, Freeman WJ, et al. Breathing as a Fundamental Rhythm of Brain Function. Frontiers in Neural Circuits. 2017; 10: 115.

[45] Tort ABL, Brankačk J, Draguhn A. Respiration-Entrained Brain Rhythms Are Global but often Overlooked. Trends in Neurosciences. 2018; 41: 186-197.

[46] Fontanini A, Spano PF, Bower JM. Ketamine-Xylazine-Induced Slow $(<1.5 \mathrm{~Hz})$ Oscillations in the Rat Piriform (Olfactory) Cortex are Functionally Correlated with Respiration. The Journal of Neuroscience. 2003; 23: 7993-8001.

[47] Yanovsky Y, Ciatipis M, Draguhn A, Tort ABL, Brankačk J. Slow oscillations in the mouse hippocampus entrained by nasal respiration. The Journal of Neuroscience. 2014; 34: 5949-5964.

[48] Nguyen Chi V, Müller C, Wolfenstetter T, Yanovsky Y, Draguhn A, Tort ABL, et al. Hippocampal Respiration-Driven Rhythm Dis- 
tinct from Theta Oscillations in Awake Mice. The Journal of Neuroscience. 2016; 36: 162-177.

[49] Ito J, Roy S, Liu Y, Cao Y, Fletcher M, Lu L, et al. Whisker barrel cortex delta oscillations and gamma power in the awake mouse are linked to respiration. Nature Communications. 2014; 5: 3572.

[50] Tsanov M, Chah E, Reilly R, O'Mara SM. Respiratory cycle entrainment of septal neurons mediates the fast coupling of sniffing rate and hippocampal theta rhythm. The European Journal of Neuroscience. 2014; 39: 957-974.

[51] Lockmann AL, Laplagne DA, Leão RN, Tort AB. RespirationCoupled Rhythm in the Rat Hippocampus Independent of Theta and Slow Oscillations. Journal of Neuroscience. 2016; 36: 53385352.

[52] Lockmann ALV, Tort ABL. Nasal respiration entrains deltafrequency oscillations in the prefrontal cortex and hippocampus of rodents. Brain Structure and Function. 2018; 223: 1-3.

[53] Zhong W, Ciatipis M, Wolfenstetter T, Jessberger J, Müller C, Ponsel S, et al. Selective entrainment of gamma subbands by different slow network oscillations. Proceedings of the National Academy of Sciences of the United States of America. 2017; 114: 4519-4524.

[54] Biskamp J, Bartos M, Sauer JF. Organization of prefrontal network activity by respiration-related oscillations. Scientific Reports. 2017; 7: 45508.

[55] Moberly AH, Schreck M, Bhattarai JP, Zweifel LS, Luo W, Ma M. Olfactory inputs modulate respiration-related rhythmic activity in the prefrontal cortex and freezing behavior. Nature Communications. 2018; 9: 1528.

[56] Perl O, Ravia A, Rubinson M, Eisen A, Soroka T, Mor N, et al. Human non-olfactory cognition phase-locked with inhalation. Nature Human Behaviour. 2019; 3: 501-512.

[57] Piarulli A, Zaccaro A, Laurino M, Menicucci D, De Vito A, Bruschini L, et al. Ultra-slow mechanical stimulation of olfactory epithelium modulates consciousness by slowing cerebral rhythms in humans. Scientific Reports. 2018; 8: 6581.

[58] Grosmaitre X, Santarelli LC, Tan J, Luo M, Ma M. Dual functions of mammalian olfactory sensory neurons as odor detectors and mechanical sensors. Nature Neuroscience. 2007; 10: 348-354.

[59] Ravel N, Pager J. Respiratory patterning of the rat olfactory bulb unit activity: nasal versus tracheal breathing. Neuroscience Letters. 1990; 115: 213-218.

[60] Phillips ME, Sachdev RN, Willhite DC, Shepherd GM. Respiration drives network activity and modulates synaptic and circuit processing of lateral inhibition in the olfactory bulb. The Journal of Neuroscience. 2012; 32: 85-98.

[61] Kleinfeld D, Deschênes M, Wang F, Moore JD. More than a rhythm of life: breathing as a binder of orofacial sensation. Nature Neuroscience. 2014; 17: 647-651.

[62] Yackle K, Schwarz LA, Kam K, Sorokin JM, Huguenard JR, Feldman JL, et al. Breathing control center neurons that promote arousal in mice. Science. 2017; 355: 1411-1415.
[63] Karalis N, Sirota A. Breathing coordinates limbic network dynamics underlying memory consolidation. bioRxiv. 2018. (in press)

[64] Tort ABL, Hammer M, Zhang J, Brankačk J, Draguhn A. Temporal Relations between Cortical Network Oscillations and Breathing Frequency during REM Sleep. The Journal of Neuroscience. 2021; 41: 5229-5242.

[65] Carey RM, Wachowiak M. Effect of Sniffing on the Temporal Structure of Mitral/Tufted Cell Output from the Olfactory Bulb. Journal of Neuroscience. 2011; 31: 10615-10626.

[66] McBride DW, Hamill OP. Pressure-clamp technique for measurement of the relaxation kinetics of mechanosensitive channels. Trends in Neurosciences. 1993; 16: 341-345.

[67] Maroto R, Kurosky A, Hamill OP. Mechanosensitive Ca(2+) permeant cation channels in human prostate tumor cells. Channels. 2012; 6: 290-307.

[68] Lewis AH, Cui AF, McDonald MF, Grandl J. Transduction of Repetitive Mechanical Stimuli by Piezo1 and Piezo2 Ion Channels. Cell Reports. 2017; 19: 2572-2585.

[69] Yasuma F, Hayano J. Respiratory sinus arrhythmia: why does the heartbeat synchronize with respiratory rhythm? Chest. 2004; 125: 683-690.

[70] Mather M, Thayer J. How heart rate variability affects emotion regulation brain networks. Current Opinion in Behavioral Sciences. 2018; 19: 98-104.

[71] Frysinger RC, Harper RM. Cardiac and respiratory correlations with unit discharge in human amygdala and hippocampus. Electroencephalography and Clinical Neurophysiology. 1989; 72: 463-470.

[72] Sobel N, Prabhakaran V, Hartley CA, Desmond JE, Zhao Z, Glover $\mathrm{GH}$, et al. Odorant-induced and sniff-induced activation in the cerebellum of the human. The Journal of Neuroscience. 1998; 18: 8990-9001.

[73] Cao Y, Liu Y, Jaeger D, Heck DH. Cerebellar Purkinje Cells Generate Highly Correlated Spontaneous Slow-Rate Fluctuations. Frontiers in Neural Circuits. 2017; 11: 67.

[74] Vinje V, Ringstad G, Lindstrøm EK, Valnes LM, Rognes ME, Eide PK, et al. Respiratory influence on cerebrospinal fluid flow - a computational study based on long-term intracranial pressure measurements. Scientific Reports. 2019; 9: 9732.

[75] Duvernoy HM, Delon S, Vannson JL. Cortical blood vessels of the human brain. Brain Research Bulletin. 1981; 7: 519-579.

[76] Heintz N. Gene expression nervous system atlas (GENSAT). Nature Neuroscience. 2004; 7: 483.

[77] Chesler AT, Szczot M, Bharucha-Goebel D, Čeko M, Donkervoort S, Laubacher $\mathrm{C}$, et al. The Role of PIEZO2 in Human Mechanosensation. New England Journal of Medicine. 2016; 375: 1355-1364.

[78] Buzsaki G. Rhythms of the brain. Oxford University Press: Oxford. 2006. 